\title{
Tailoring steroids in the treatment of COVID-19 pneumonia assisted by CT scans: three case reports
}

Ying Su${ }^{\mathrm{a}, 1}$, Yi Han ${ }^{\mathrm{b}, 1}$, Jie Liuc ${ }^{\mathrm{c}}$, Yue Qiu ${ }^{\mathrm{a}}$, Qian Tan ${ }^{\mathrm{d}}$, Zhen Zhou ${ }^{\mathrm{e}}$, Yi-zhou Yu ${ }^{\mathrm{f}}$, Jun Chen ${ }^{\mathrm{g}}$, Maryellen L. Giger ${ }^{\mathrm{h}}$, Fleming Y. M. Lure and Zhe Luo ${ }^{\mathrm{a}, *}$

a Department of Critical Care Medicine, Zhongshan Hospital, Fudan University, Shanghai, P.R. China

${ }^{\mathrm{b}}$ Department of Emergency, Zhongshan Hospital, Fudan University, Shanghai, P.R. China

${ }^{\mathrm{c}}$ Department of Respiratory Medicine, Zhongshan Hospital, Fudan University, Shanghai, P.R. China

${ }^{\mathrm{d}}$ Department of Lung Cancer, Tianjin Medical University Cancer Institute and Hospital, Tianjin Lung Cancer Center, P.R. China

${ }^{\mathrm{e}}$ School of Electronics Engineering and Computer Science, Peking University, Peking, P.R. China

${ }^{\mathrm{f}}$ Department of Computer Science, The University of Hong Kong, Hong Kong, P.R. China

${ }^{\mathrm{g}}$ Department of Radiology, Renmin Hospital of Wuhan University, Wuhan, P.R. China

${ }^{\mathrm{h}}$ Department of Radiology, University of Chicago, Chicago, IL, USA

${ }^{i}$ MS Technologies Corp, Rockville, MD 20850; Shenzhen Smart Imaging Healthcare Co,

Shenzhen, China

Received 12 May 2020

Revised 13 June 2020

Accepted 19 June 2020

\begin{abstract}
In this article, we analyze and report cases of three patients who were admitted to Renmin Hospital, Wuhan University, China, for treating COVID-19 pneumonia in February 2020 and were unresponsive to initial treatment of steroids. They were then received titrated steroids treatment based on the assessment of computed tomography (CT) images augmented and analyzed with the artificial intelligence (AI) tool and output. Three patients were finally recovered and discharged. The result indicated that sufficient steroids may be effective in treating the COVID-19 patients after frequent evaluation and timely adjustment according to the disease severity assessed based on the quantitative analysis of the images of serial CT scans.
\end{abstract}

Keywords: Coronavirus, pneumonia, treating COVID-19 patients, steroids, computerized tomography, image analysis using artificial intelligence

\section{Introduction}

Since December 2019, an outbreak of a novel coronavirus infected pneumonia (COVID-19) has led worldwide panic. As of April 16th, more than 2,011,725 cases and 131,150 deaths were confirmed globally. Furthermore, it has been reported that a 28-day mortality of critically ill patients with COVID19 pneumonia was at $61.5 \%$ (32/52), even with advanced organ supporting techniques [1]. Notably, the

\footnotetext{
${ }^{1}$ Ying Su and Yi Han contributed equally to this article.

${ }^{*}$ Corresponding author: Zhe Luo, Department of Critical Care Medicine, Zhongshan Hospital, Fudan University, Shanghai, P.R. China. E-mail: luo.zhe@zs-hospital.sh.cn.
} 
presentation of overwhelming inflammation has been considered as the dominant mechanism accounting for the worsening of COVID-19 pneumonia. This infers that steroids may be a recommended prescription for reducing the inflammatory response, and thus a potential strategy for reversing progression. However, whether to use it in viral pneumonia is widely argued and even objected to in COVID-19 pneumonia [2].

In this article, we analyze and report three COVID-19 pneumonia patients who were unresponsive to initial treatment of steroids but were ultimately cured through a tailored strategy. This strategy incorporated the use of computerized tomography (CT) images analyzed by an artificial intelligence (AI) system to monitor progression and facilitate clinical decision-making.

\section{Materials and methods}

We retrospectively identified three patients who were admitted into the intensive care unit at Renmin Hospital, Wuhan University (Hubei province, China), for COVID-19 pneumonia in February 2020, and were attended to by a medical rescuing team from Zhongshan Hospital, Fudan University (Shanghai, China). Inclusion criteria required that patients were diagnosed as COVID-19 by Reverse Transcription-Polymerase Chain Reaction (RT-PCR) and were unresponsive to initial steroid treatment. The therapeutic details during hospital stay were presented in Figs. 1-3.

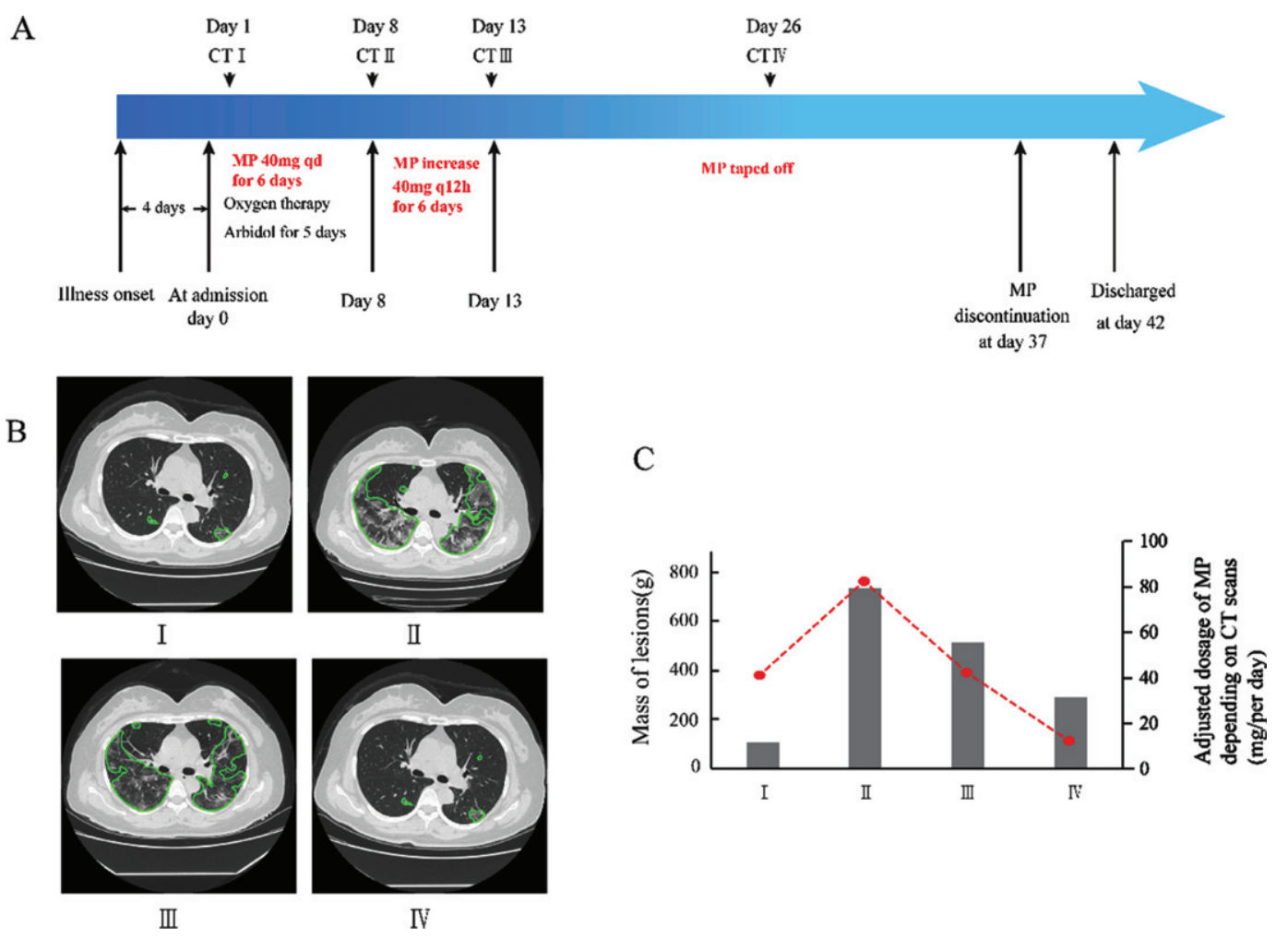

Fig. 1. Timeline of disease course of case 1. A. Therapeutic details according to days from illness onset and days from hospital admission. B. Chest CT images of the patient at 4 stages. Chest CT scans were performed on hospital day 1 (I), day 8 (II), day 13 (III), and day 26 (IV), respectively. Areas within green lines indicate the lesions of lungs analyzed by AI system. C. The changes of mass of lesions at 4 stages and the adjusted dosage of MP (mg/per day) depending on CT scans. Mass was calculated as: $\mathrm{M}=\mathrm{V} *(\mathrm{~A}+1000) / 1000$, where $\mathrm{M}$ is mass in $\mathrm{g}, \mathrm{V}$ is volume in $\mathrm{cm}^{3}$, and $\mathrm{A}$ is mean attenuation in Hounsfield Unit (HU, reflects the degree of X-ray absorption of the tissue). MP, Methylprednisolone. 
A

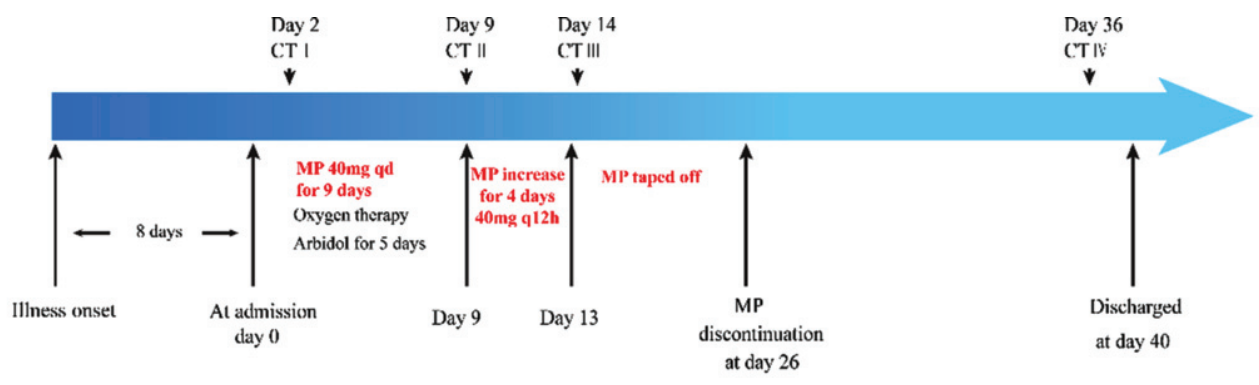

B

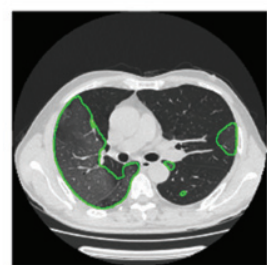

1

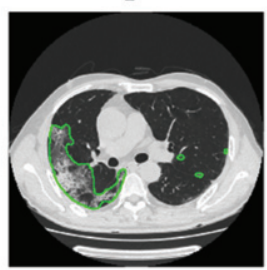

III

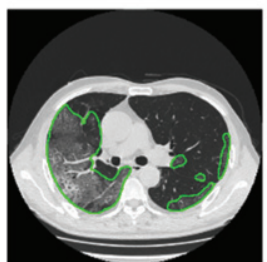

॥

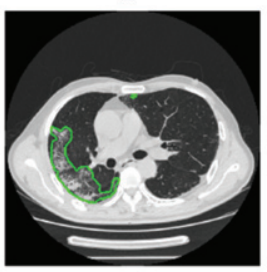

IV

$\mathrm{C}$

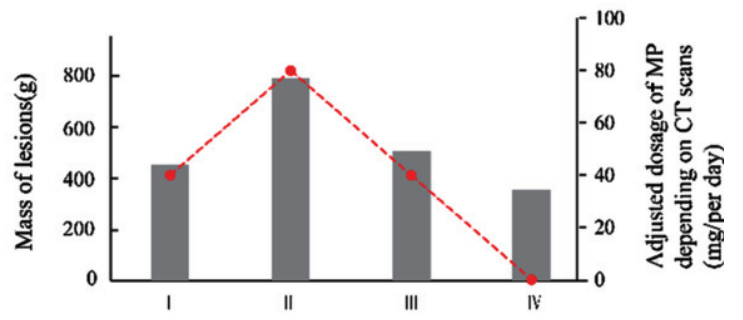

Fig. 2. Timeline of disease course of case 2. A. Therapeutic details according to days from illness onset and days from hospital admission. B. Chest CT images of the patient at 4 stages. Chest CT scans were performed on hospital day 2 (I), hospital day 9 (II), day 14 (III), and day 36 (IV), respectively. Areas within green lines indicate the lesions of lungs analyzed by AI system. C. The changes of mass of lesions at 4 stages and the adjusted dosage of MP (mg/per day) depending on CT scans. Mass was calculated as: $\mathrm{M}=\mathrm{V} *(\mathrm{~A}+1000) / 1000$, where $\mathrm{M}$ is mass in $\mathrm{g}, \mathrm{V}$ is volume in $\mathrm{cm}^{3}$, and $\mathrm{A}$ is mean attenuation in Hounsfield Unit (HU, reflects the degree of X-ray absorption of the tissue). MP, Methylprednisolone.

An automatic pneumonia analysis system, Dr. Wise@Pneumonia (version 1.0, Beijing Deepwise \& League of Ph.D Technology Co. LTD), was used to segment pneumonia regions in CT images and calculate corresponding quantitative measurements of volume, CT value, location, and mass, and subsequently quantify the level of COVID involvement to facilitate analysis and decision-making during treatment. The system is built based on deep neural networks, where two major modules were designed to ensure the effectiveness and efficiency in pneumonia detection, and voxel segmentation. First, an MVP-Net [3] was used to detect bounding boxes of pneumonia findings in CT images. Attention mechanism and 3D context module were applied to enhance the detection sensitivity, and multiple binary symptom classifiers were trained to discriminate the consolidation, ground-glass opacity, nodule and so forth. In our internal dataset, the MVP-Net detected $90.1 \%$ of consolidations, $90.3 \%$ of ground glass opacities, and $91.5 \%$ of pulmonary nodules. Then U-net is used to classify each voxel within the 3D cube into lesion or non-lesion class [4]. The trained system was used on all three cases in these reports.

\section{Results}

\subsection{Case 1}

A 54-year-old female complained of fever and chills for 4 days accompanied with dyspnea and diarrhea. Chest CT scan on hospital day 1showed multifocal ground glass opacities (GGO) and infiltrates located on bilateral lungs (Fig. 1). On admission, she received supplemental oxygen via mask and 
A

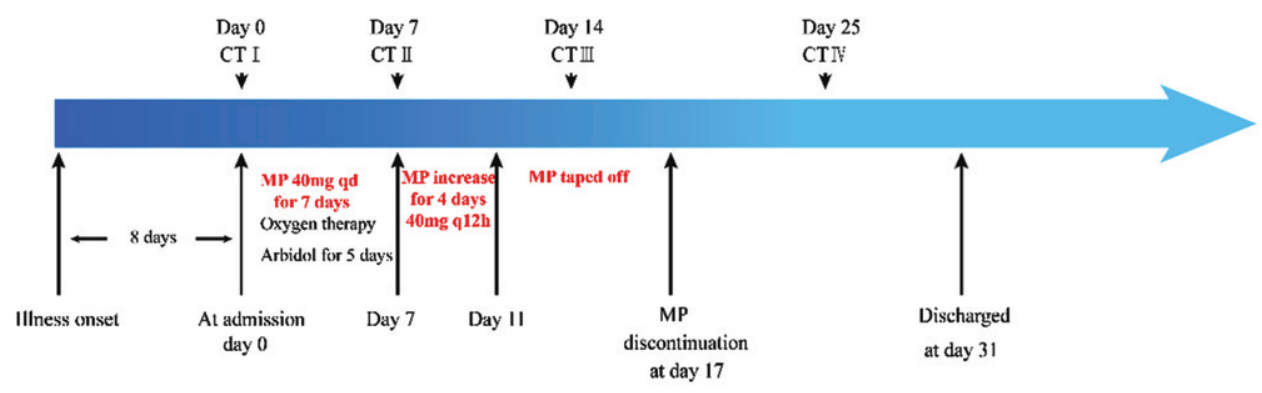

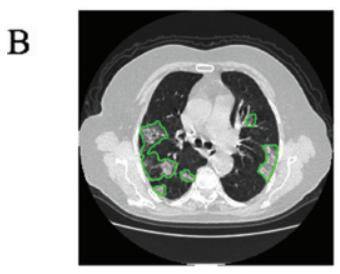

I

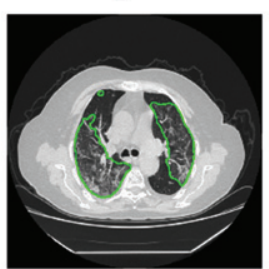

III

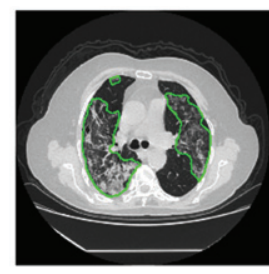

II

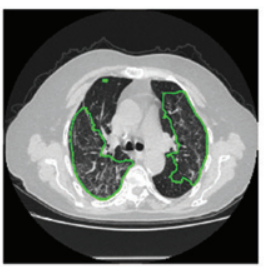

IV

C

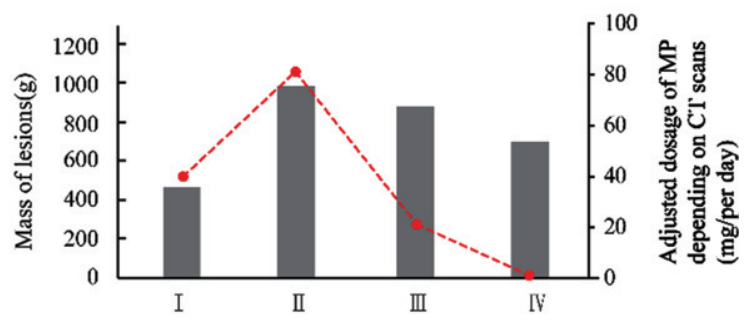

Fig. 3. Timeline of disease course of case 3. A. Therapeutic details according to days from illness onset and days from hospital admission. B. Chest CT images of the patient at 4 stages. Chest CT scans were performed on day of admission (I), hospital day 7 (II), day 14 (III), and day 25 (IV), respectively. Areas within green lines indicate the lesions of lungs analyzed by AI system. C. The changes of mass of lesions at 4 stages and the adjusted dosage of MP (mg/per day) depending on $\mathrm{CT}$ scans. Mass was calculated as: $\mathrm{M}=\mathrm{V} *(\mathrm{~A}+1000) / 1000$, where $\mathrm{M}$ is mass in $\mathrm{g}$, $\mathrm{V}$ is volume in $\mathrm{cm}^{3}$, and $\mathrm{A}$ is mean attenuation in Hounsfield Unit (HU, reflects the degree of X-ray absorption of the tissue). MP, Methylprednisolone.

arbidol. At day 2, because of progressive dyspnea, immune globulin and low-dose Methylprednisolone (MP) at $40 \mathrm{mg}$ qd $(0.67 \mathrm{mg} / \mathrm{kg} / \mathrm{d})$ for 6 days were added (Fig. 1A), and the patient's clinical condition seemed under control. On hospital day 8 , her temperature increased to $38.5^{\circ} \mathrm{C}$ again with worsening dyspnea. A follow-up CT scan confirmed the deterioration of pneumonia with remarkably enlarged diffused bilateral opacities (Fig. 1B-II), and the mass of lesion presented a sharp increase (Fig. 1C). Mass of lesion was calculated as: $\mathrm{M}=\mathrm{V} *(\mathrm{~A}+1000) / 1000$, where $\mathrm{M}$ is mass in $\mathrm{g}$, $\mathrm{V}$ is volume in $\mathrm{cm}^{3}$, and A is mean attenuation in Hounsfield Unit (HU, reflects the degree of X-ray absorption of the tissue) $[5,6]$. Subsequently, the flow in her mask piece was increased to $10 \mathrm{~L} / \mathrm{min}$, and MP dosage to $40 \mathrm{mg}$ every 12 hours $(1.33 \mathrm{mg} / \mathrm{kg} / \mathrm{d})$ for the next 6 days, while other therapeutic regimens remained unchanged. The patient's clinical condition improved, including body temperature and oxygenation, and MP was then tapered off. The CT scan on day 13 and day 26 showed bilateral diffuse opacities and infiltrates dissipated gradually (Fig. 1B-III and IV; 1C). Finally, the patient was discharged from hospital on day 42 .

\subsection{Case 2}

A 69-year-old male presented to fever clinic with a history of 8-day of fever and 2-day of dyspnea. The chest CT scan on hospital day 2 showed bilateral ground glass opacities (Fig. 2B-I). Although the 
patient was accommodated with oxygenation support, arbidol, immunoglobulin, and MP $40 \mathrm{mg} / \mathrm{day}$ $(0.62 \mathrm{mg} / \mathrm{kg} / \mathrm{d})$ (Fig. 2A) on admission, his weakness and dyspnea persisted. On hospital day 9 , his body temperature rose to $38.2^{\circ} \mathrm{C}$, follow-up CT showed obvious peripheral infiltration on the right lung (Fig. 2B-II), and the mass of lesion increased (Fig. 2C). The patient's respiratory status deteriorated even with high gas flow via mask piece. Therefore, he was treated initially with high flow nasal cannula (HFNC) oxygenation, with a flow rate of $60 \mathrm{~L} / \mathrm{min}, 80 \% \mathrm{FiO}_{2}$. Meanwhile, MP was added to $40 \mathrm{mg}$ every 12 hours $(1.23 \mathrm{mg} / \mathrm{kg} / \mathrm{d})$ for the next 4 days. His temperature returned to normal immediately, and oxygenation also gradually elevated. At hospital day 14, CT image showed previous infiltration partially dissipated with small patchy consolidation (Fig. 2B-III), and the mass of involvement were also reduced markedly (Fig. 2C). Then, MP was tapered off. At day 36, CT images showed previous infiltration significantly dissipated and the consolidation was partially absorbed (Fig. 2B-IV), and the patient was discharged on day 40.

\subsection{Case 3}

A 75-year-old female initially complained of fever and dyspnea accompanying with fatigue. The pre-admission chest CT scan showed multifocal GGO and infiltrates on bilateral lungs (Fig. 3B-I). On admission, supplemental oxygen via mask, arbidol, immunoglobulin, and low-dose MP $40 \mathrm{mg} / \mathrm{day}$ $(0.56 \mathrm{mg} / \mathrm{kg} / \mathrm{d})$ was given. However, the patient's clinical condition was not resolved. On hospital day 7, a follow-up CT scan revealed enlarged diffused bilateral opacities and sharp increased lesion mass of COVID involvement (Fig. 3C). Subsequently, the gas flow and MP dosage were increased to $40 \mathrm{mg}$ every 12 hours $(1.11 \mathrm{mg} / \mathrm{kg} / \mathrm{d})$ for the next 4 days. The dyspnea and oxygenation were markedly relieved, and thus, MP was tapered off. Although the CT scans on day 14 showed only slightlydissipated bilateral diffuse opacities and infiltrates, and the mass of lesion increased (Fig. 3B-III, IV, 3C), the mass of lesions was reduced markedly (Fig. 3C). Finally, as CT scans demonstrated dissipated bilateral diffuse opacities and infiltrates, the patient was discharged on day 31 .

\section{Discussion}

Much debate exists over the effectiveness of steroids on COVID-19 patients. As shown in our three case reports, although all the cases had MP prescribed at an initial dosage of $40 \mathrm{mg}$ once every day (average dose $0.62 \mathrm{mg} / \mathrm{kg} / \mathrm{d}$ ) and all empirically failed to prevent progression of intrapulmonary inflammation, a significant and steady improvement was observed after simply increasing the MP dosage. Thus, we note that tailoring a strategy with serial CT monitoring might facilitate efficiency of steroids.

The application of steroids in the treatment of pneumonia can be traced back to more than 60 years ago [7]. It is often used in community acquired pneumonia (CAP) [8-11] and acute respiratory distress syndrome (ARDS) [12-14], exhibiting many positive results. Note that short-term low to moderate steroids, of even $1 \mathrm{mg} / \mathrm{kg} / \mathrm{d}$ MP for 2 weeks initially, could show capacity in inhibiting inflammation and improving the prognosis of ARDS. However, in viral pneumonia, studies often showed conflicting results, which perplex clinicians during decision-making. First, patients involved in these clinical studies were characterized by different symptoms and degree of severity, which might have introduced bias into the result. Second, uncertainty of dosage of steroids always disturbs clinicians. Research regarding SARS claimed that low dose of steroids $(<500 \mathrm{mg} / \mathrm{d}$, all dosage mentioned in this article were equivalent dosage of MP) for patients with mildest symptoms [15-18], or high dose ( $\geq 500 \mathrm{mg} / \mathrm{d})$ for patients with severe hypothermia $[15,19]$ seemed effective in both improving symptoms and outcome, although more comorbidities occurred with the higher dose [20,21]. In studies of influenza 
A and Middle East respiratory syndrome (MERS), MP dosages varied from 5 to $180 \mathrm{mg} / \mathrm{d}$, with no patients having exhibited a reduction in severity or mortality [22-33]. Third, there is no specific or quantitative indicator guiding the timing of application, adjustment, or withdrawal of steroids. In this report, considering lack of high-quality research, we tailored steroids therapy based on frequent CT-based evaluations.

According to recent publications on CT image in COVID-19 pneumonia, we expected to find patchy/punctate GGO, ground glass nodules (GGN), patchy consolidation, fibrous stripes and irregular solid nodules [34]. Note that abnormal pulmonary CT manifestations can occur even in asymptomatic patients. The COVID-19 presentation can rapidly develop into diffuse ground-glass shadows or consolidation within 1-3 weeks after the onset of symptoms, reaching a peak at approximately 2 weeks after onset and correlating well with disease severity [35]. With the advent of the "imaging artificial intelligence pneumonia auxiliary diagnosis system," which measured the volume and mass of COVID involvement, we were able to quantitatively and objectively evaluate CT imaging changes, incorporating information beyond that observed by visual evaluation (Fig. 3B-III, 3C and D). Using algorithm similar to that of computing lung nodule mass, we explored the possibility of assessing severity or treatment efficacy of COVID-19 patients. Thus, we speculate that such technology may have the potential to provide specific indicators for precise management of steroids, although a large-scale clinical trial is necessary for wide-spread adoption.

In conclusion, the cases of three patients analyzed and reported in this article demonstrate that steroids can be effective in the treatment of COVID-19 pneumonia when based on a tailoring strategy incorporating quantitative analysis of serial CT images with the advanced AI. Specifically, CT images analyzed by the AI system may facilitate efficacy of steroid therapy in treating COVID-19 patients. In the future, the relationship between the data provided by AI system and the treatment effect will be further analyzed in a large population study.

\section{Declaration of interests}

The authors declare that they have no competing interests.

\section{Acknowledgments}

Not applicable.

\section{References}

[1] C. Huang, Y. Wang, X. Li, et al., Clinical features of patients infected with 2019 novel coronavirus in Wuhan, China, Lancet (London, England) 395(10223) (2020), 497-506.

[2] C.D. Russell, J.E. Millar and J.K. Baillie, Clinical evidence does not support corticosteroid treatment for 2019-nCoV lung injury, The Lancet 395(10223) (2020), 473-475.

[3] Z. Li, S. Zhang, J. Zhang, et al., MVP-Net: Multi-view FPN with Position-Aware Attention for Deep Universal Lesion Detection. In: 2019; Cham: Springer International Publishing; (2019), 13-21.

[4] O. Ronneberger, P. Fischer and T. Brox, U-Net: Convolutional Networks for Biomedical Image Segmentation. In: 2015; Cham: Springer International Publishing; (2015), 234-241.

[5] B. de Hoop, H. Gietema, S. van de Vorst, et al., Pulmonary ground-glass nodules: increase in mass as an early indicator of growth, Radiology 255(1) (2010), 199-206.

[6] Y.S. Song, C.M. Park, S.J. Park, et al., Volume and mass doubling times of persistent pulmonary subsolid nodules detected in patients without known malignancy, Radiology 273(1) (2014), 276-284.

[7] P.S. Blom and P. Van Leeuwen, Recovery in a case of fulminating influenza virus pneumonia following a rapid intravenous administration of hydrocortisone, Nederlands Tijdschrift Voor Geneeskunde 103(3) (1959), 109-110. 
[8] A. Torres, O. Sibila, M. Ferrer, et al., Effect of corticosteroids on treatment failure among hospitalized patients with severe community-acquired pneumonia and high inflammatory response: a randomized clinical trial, JAMA 313(7) (2015), 677-686.

[9] Q.U. Nawab, E. Golden, M. Confalonieri, et al., Corticosteroid treatment in severe community-acquired pneumonia: duration of treatment affects control of systemic inflammation and clinical improvement, Intensive Care Med 37(9) (2011), 1553-1554.

[10] S.C. Meijvis, H. Hardeman, H.H. Remmelts, et al., Dexamethasone and length of hospital stay in patients with community-acquired pneumonia: a randomised, double-blind, placebo-controlled trial, Lancet 377(9782) (2011), 2023-2030.

[11] F.E. Lee, E.E. Walsh and A.R. Falsey, The effect of steroid use in hospitalized adults with respiratory syncytial virus-related illness, Chest 140(5) (2011), 1155-1161.

[12] K.P. Steinberg, L.D. Hudson, R.B. Goodman, et al., Efficacy and safety of corticosteroids for persistent acute respiratory distress syndrome, $N$ Engl J Med 354(16) (2006), 1671-1684.

[13] G.U. Meduri, E. Golden, A.X. Freire, et al., Methylprednisolone infusion in early severe ARDS: results of a randomized controlled trial, Chest 131(4) (2007), 954-963.

[14] M. Confalonieri, R. Urbino, A. Potena, et al., Hydrocortisone infusion for severe community-acquired pneumonia: a preliminary randomized study, Am J Respir Crit Med 171(3) (2005), 242-248.

[15] L.Y. Yam, A.C. Lau, F.Y. Lai, et al., Corticosteroid treatment of severe acute respiratory syndrome in Hong Kong, The Journal of Infection 54(1) (2007), 28-39.

[16] J.C. Ho, G.C. Ooi, T.Y. Mok, et al., High-dose pulse versus nonpulse corticosteroid regimens in severe acute respiratory syndrome, Am J Respir Crit Care Med 168(12) (2003), 1449-1456.

[17] T.W. Auyeung, J.S. Lee, W.K. Lai, et al., The use of corticosteroid as treatment in SARS was associated with adverse outcomes: a retrospective cohort study, The Journal of Infection 51(2) (2005), 98-102.

[18] N. Lee, Allen Chan KC, D.S. Hui, et al., Effects of early corticosteroid treatment on plasma SARS-associated Coronavirus RNA concentrations in adult patients, Journal of Clinical Virology 31(4) (2004), 304-309.

[19] R.C. Chen, X.P. Tang, S.Y. Tan, et al., Treatment of severe acute respiratory syndrome with glucosteroids: the Guangzhou experience, Chest 129(6) (2006), 1441-1452.

[20] J.J. Sung, A. Wu, G.M. Joynt, et al., Severe acute respiratory syndrome: report of treatment and outcome after a major outbreak, Thorax 59(5) (2004), 414-420.

[21] R. Zhao, H. Wang, X. Wang and F. Feng, Steroid therapy and the risk of osteonecrosis in SARS patients: a dose-response meta-analysis, Osteoporosis International 28(3) (2017), 1027-1034.

[22] A.A. Boudreault, H. Xie, W. Leisenring, et al., Impact of corticosteroid treatment and antiviral therapy on clinical outcomes in hematopoietic cell transplant patients infected with influenza virus, Biology of Blood and Marrow Transplantation 17(7) (2011), 979-986.

[23] C. Brun-Buisson, J.C. Richard, A. Mercat, et al., Early corticosteroids in severe influenza A/H1N1 pneumonia and acute respiratory distress syndrome, Am J Respir Crit Care Med 183(9) (2011), 1200-1206.

[24] K. Han, H. Ma, X. An, et al., Early use of glucocorticoids was a risk factor for critical disease and death from pH1N1 infection, Clinical Infectious Diseases 53(4) (2011), 326-333.

[25] R. Linko, V. Pettila, E. Ruokonen, et al., Corticosteroid therapy in intensive care unit patients with PCR-confirmed influenza A(H1N1) infection in Finland, Acta Anaesthesiologica Scandinavica 55(8) (2011), 971-979.

[26] M. Delgado-Rodriguez, J. Castilla, P. Godoy, et al., Prognosis of hospitalized patients with 2009 H1N1 influenza in Spain: influence of neuraminidase inhibitors, The Journal of Antimicrobial Chemotherapy 67(7) (2012), 1739-1745.

[27] M. Al-Busaidi, K. Al Maamari, B. Al'Adawi, et al., Pandemic Influenza A H1N1 in Oman: Epidemiology, Clinical Features, and Outcome of Patients Admitted to Sultan Qaboos University Hospital in 2009, Oman Medical Journal 31(4) (2016), 290-297.

[28] B. Cao, H. Gao, B. Zhou, et al., Adjuvant Corticosteroid Treatment in Adults With Influenza A (H7N9) Viral Pneumonia, Crit Care Med 44(6) (2016), e318-328.

[29] J.W. Delaney, R. Pinto, J. Long, et al., The influence of corticosteroid treatment on the outcome of influenza A(H1N1pdm09)-related critical illness, Crit Care 20 (2016), 75.

[30] S.F. Huang, C.P. Fung, D.W. Perng and F.D. Wang, Effects of corticosteroid and neuraminidase inhibitors on survival in patients with respiratory distress induced by influenza virus, Journal of Microbiology, Immunology, and Infection 50(5) (2017), 586-594.

[31] H. Li, S.G. Yang, L. Gu, et al., Effect of low-to-moderate-dose corticosteroids on mortality of hospitalized adolescents and adults with influenza A(H1N1)pdm09 viral pneumonia, Influenza and Other Respiratory Viruses 11(4) (2017), $345-354$. 
[32] G. Moreno, A. Rodriguez, L.F. Reyes, et al., Corticosteroid treatment in critically ill patients with severe influenza pneumonia: a propensity score matching study, Intensive Care Med 44(9) (2018), 1470-1482.

[33] Y.M. Arabi, Y. Mandourah, F. Al-Hameed, et al., Corticosteroid Therapy for Critically Ill Patients with Middle East Respiratory Syndrome, Am J Respir Crit Care Med 197(6) (2018), 757-767.

[34] Q. Zheng, Y. Lu, F. Lure, et al., Clinical and radiological features of novel coronavirus pneumonia, J Xray Sci Technol 28(3) (2020), 391-404.

[35] Y. Pan, H. Guan, S. Zhou, et al., Initial CT findings and temporal changes in patients with the novel coronavirus pneumonia (2019-nCoV): a study of 63 patients in Wuhan, China, Eur Radiol 30(6) (2020), 3306-3309. 